\title{
Edge states on graphene ribbon in magnetic field: interplay between Dirac and ferromagnetic-like gaps
}

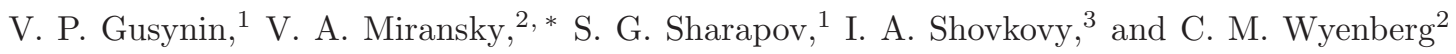 \\ ${ }^{1}$ Bogolyubov Institute for Theoretical Physics, 03680, Kiev, Ukraine \\ ${ }^{2}$ Department of Applied Mathematics, University of Western Ontario, London, Ontario, Canada N6A 5Br \\ ${ }^{3}$ School of Applied Arts and Sciences, Arizona State University, Mesa, Arizona 85212, USA
}

(Dated: November 13, 2018)

\begin{abstract}
By combining analytic and numerical methods, edge states on a finite width graphene ribbon in a magnetic field are studied in the framework of low-energy effective theory that takes into account the possibility of quantum Hall ferromagnetism (QHF) gaps and dynamically generated Dirac-like masses. The analysis is done for graphene ribbons with both zigzag and armchair edges. The characteristic features of the spectrum of the edge states in both these cases are described. In particular, the conditions for the existence of the gapless edge states are established. Implications of these results for the interpretation of recent experiments are discussed.
\end{abstract}

PACS numbers: 73.43.Cd, 71.70.Di, 73.22.Gk

\section{INTRODUCTION}

Graphene is a remarkable system with many unusual properties that was created for the first time only a few years ago $\stackrel{1}{=}$ (For reviews on graphene see, for example, Refs. 2, 3, 4,5.) One of such properties is an unconventional quantum Hall effect (QHE). Instead of the ordinary QHE, expected in the case of 2-dimensional non-relativistic electron systems, an anomalous quantization is observed in graphene..$\underline{6}$ The observation appears to be in perfect agreement with the theoretical predictions, $\underline{\underline{8}, \underline{9}, \underline{10}}$ stating that the QHE plateaus should occur at filling factors $\nu= \pm 4(|n|+1 / 2)$ where $n$ is an integer. This anomalous quantization is a direct outcome of the relativistic-like nature of the low-energy quasiparticles in graphene described by a Dirac theory with an internal $U(4)$ symmetry $\underline{11,12}$

It is the $U(4)$ symmetry, operating in the spin and sublattice-valley spaces, that is responsible for the 4 -fold degeneracy of the Landau energy levels and for the overall factor 4 in the filling factors of the observed plateaus, $\nu= \pm 4(|n|+1 / 2)$. Strictly speaking, the $U(4)$ symmetry is not exact but broken down to a smaller $U(2)_{+} \times U(2)_{-}$ symmetry group by the Zeeman term. The latter symmetry operates in the sublattice-valley space and does not mix spin-up $(s=+)$ and spin-down $(s=-)$ states. When the magnetic field is not too strong, a relatively small Zeeman term does not affect the observable QHE in a qualitative way.

When the magnetic field becomes sufficiently strong, the QHE plateaus $\nu=0, \pm 1, \pm 4$ are observed $\stackrel{13,14,15,16}{2}$ This suggests that the 4-fold degeneracy of the Landau levels is lifted. The new plateaus may be explained by one of the following seemingly different theoretical scenarios. (i) Quantum Hall ferromagnetism (QHF) $17,18,19,20,21$, which is connected with the theory of exchange-driven spin splitting of Landau levels $\stackrel{22}{\underline{2}}$ The QHF order parameters are densities of the conserved charges connected with three diagonal generators of the non-abelian subgroup $S U(4) \subset U(4)$ (the dynamics of a Zeeman spin splitting enhancement considered in Ref. 23 is intimately connected with the QHF). (ii) The magnetic catalysis (MC) scenario $\underline{24,25,26,27}$ that is based on the phenomenon of an enhancement of the density of states in infrared by a magnetic field, which catalyzes electron-hole pairing (leading to excitonic condensates) in relativistic-like systems $\stackrel{28,29,30}{2}$. This scenario invokes electron-hole pairing and excitonic condensates to produce dynamically generated Dirac-like masses in the low-energy theory.

Recently, by analyzing a gap equation for the propagator of Dirac quasiparticles, it has been found in Refs. 31,32 that the QHF and MC order parameters necessarily coexist. As will be shown in the present paper, this feature could have important consequences for the dynamics of edge states in the QHE in graphene.

The study of edge states is of general interest because such states provide a deeper insight into the quantum Hall effect $\stackrel{33}{\underline{3}}$ Currently there exist many studies of edge states in graphene-like systems $\underline{10,23,34,35,36,37,38,39,40,41,42,43}$ In Refs. 23. 38, it was found that in the presence of a magnetic field there may exist gapless modes of such states and they should play an important role in charge transport of graphene near the Dirac neutral point. The gapless modes were shown to appear when the lowest Landau level (LLL) was split by a spin gap, or in other words, by enhanced Zeeman splitting leading to the $\nu=0$ plateau. Such gapless states were absent, though, in the case of a Dirac mass gap. $\frac{15,23}{12}$

While the presence of the gapless edge states should make graphene a so-called quantum Hall metal, their absence should make it an insulator $\frac{15,23}{2}$ The actual temperature dependence of the longitudinal resistivity at the $\nu=0$ plateau in Refs. 13, 15 is consistent with the metal type. Thus, it was argued that the origin of the $\nu=0$ plateau is connected with the enhanced spin (ferromagnetic) splitting of the LLL 15,23 
The conclusion of Ref. 15, 23 regarding the origin of the $\nu=0$ plateau does not appear to be universal however. The recent data from Ref. 16 reveal a clear plateau at $\nu=0$, but the temperature dependence of the diagonal component of the resistivity signals a crossover to an insulating state in high fields. This does not seem to support the existence of gapless edge states. So, one may ask whether there is indeed a Dirac-type mass gap and no spin gap in the device studied in Ref. 16?

Motivated by this question and a theoretical analysis in Refs. 31,32, the spectrum of edge states has been recently studied under the assumption that the removal of sublattice and spin degeneracies in graphene in a strong magnetic field is connected with the generation of both Dirac masses and spin gaps $\underline{42}$ The main result of that work was establishing a criterion for the existence of the gapless edge states in the QHE in graphene on a half-plane with a zigzag or armchair edge. It was concluded that the controversy between the experimental results in Refs. 15 and 16 may reflect more rich and complicated dynamics in the QHE in graphene than those considered in the QHF and MC scenarios.

In the present work, we extend the analysis in Ref. 42 to the case of a finite width graphene ribbon. The characteristic feature in our approach is combining analytic and numerical methods in the analysis of the edge states. This allows to describe the main features of the quasiparticle spectrum and, in particular, to extend the criterion obtained in Ref. 42 to a more complicated and interesting case of a graphene sample with two boundaries, which in turn yields a deeper insight in physics behind this criterion.

The paper is organized as follows. In Sec. III we discuss the low-energy field-theoretical model of graphene with $\mathrm{MC}$ and QHF dynamical order parameters used in the rest of the paper. An overview of the general formalism for studying edge states of a finite width graphene ribbon in an external magnetic field is given in Sec. III] The numerical analysis in the case of zigzag and armchair edges is presented in Sec. IV and $\nabla$ respectively. The discussion of the main results and their experimental implications are given in Sec. VI.

\section{QHF AND MC ORDER PARAMETERS IN GRAPHENE}

For convenience of the analysis of the edge state, the chiral representation of the Dirac matrices will be used here (see, for example, Ref. 5 ),

$$
\begin{aligned}
& \gamma^{0}=\tilde{\tau}^{1} \otimes \tau^{0}=\left(\begin{array}{ll}
0 & I \\
I & 0
\end{array}\right), \quad \gamma^{i}=-i \tilde{\tau}^{2} \otimes \tau^{i}=\left(\begin{array}{cc}
0 & -\tau^{i} \\
\tau^{i} & 0
\end{array}\right), \\
& \gamma_{5} \equiv i \gamma^{0} \gamma^{1} \gamma^{2} \gamma^{3}=\tilde{\tau}^{3} \otimes \tau^{0}=\left(\begin{array}{cc}
I & 0 \\
0 & -I
\end{array}\right),
\end{aligned}
$$

where $\tilde{\tau}^{i}, \tau^{i}$ are Pauli matrices and $\tau^{0}$ is the $2 \times 2$ unit matrix. The spinor field of Dirac quasiparticles $\Psi^{s T}=$ $\left(\psi_{K_{+} A}^{s}, \psi_{K_{+} B}^{s}, \psi_{K_{-} B}^{s}, \psi_{K_{-} A}^{s}\right)$ combines the Bloch states on two valleys $\left(K_{+}\right.$and $\left.K_{-}\right)$and two sublattices $(A$ and $B)$, $s$ is the spin index. The QHF order parameters are the spin density $\left\langle\Psi^{\dagger} P_{s} \Psi\right\rangle$ and the pseudospin density $\left\langle\Psi^{\dagger} \gamma^{5} P_{s} \Psi\right\rangle$, with $P_{ \pm}=\left(1 \pm \sigma^{3}\right) / 2$ being projectors on states with spin directed along $(+)$ and opposite $(-)$ the magnetic field. These order parameters are related to the chemical potentials $\mu_{s}$ and $\tilde{\mu}_{s}$, respectively. On the other hand, the MC order parameter is the vacuum expectation value of the Dirac mass term $\left\langle\bar{\Psi} \gamma^{3} P_{s} \Psi\right\rangle$ associated with the conventional Dirac mass $\tilde{\Delta}_{s}$ (here $\bar{\Psi}=\Psi^{\dagger} \gamma^{0}$ ).

Recently, a unifying approach, combining and augmenting both QHF and MC mechanisms, was proposed in Refs. 31 , 32. By analyzing the gap equation with a local Coulomb interaction and using a multi-parameter variational ansatz for the quasiparticle propagator, it was found that (i) the new MC order parameter $\left\langle\bar{\Psi} \gamma^{3} \gamma^{5} P_{s} \Psi\right\rangle$, related to a Dirac mass $\Delta_{s}$ that breaks time reversal symmetry $\underline{\underline{12}}$, has to be added, and (ii) the QHF and MC order parameters necessarily coexist. (Let us emphasize that, in the presence of an external magnetic field, the time-reversal symmetry is broken and a state with the vanishing $\Delta_{s}$ is not protected by any symmetry.)

More precisely, it was shown in Refs. 31, 32 that for a fixed spin, the full inverse quasiparticle propagator takes the following general form (in the chiral representation used in this paper):

$$
i G_{s}^{-1}\left(u, u^{\prime}\right)=\left[\left(i \hbar \partial_{t}+\mu_{s}+\tilde{\mu}_{s} \gamma^{5}\right) \gamma^{0}-v_{F}(\boldsymbol{\pi} \cdot \gamma)-\tilde{\Delta}_{s} \gamma^{3}+\Delta_{s} \gamma^{3} \gamma^{5}\right] \delta^{3}\left(u-u^{\prime}\right),
$$

where $\boldsymbol{\pi}$ is the canonical momentum and the parameters $\mu_{s}, \tilde{\mu}_{s}, \Delta_{s}$, and $\tilde{\Delta}_{s}$ are determined from the gap equation. Note that the full electron chemical potentials $\mu_{ \pm}$include the Zeeman energy $\mp Z$ with

$$
Z \simeq \mu_{B} B=0.67 B[\mathrm{~T}] \mathrm{K} .
$$


By making use of the explicit form of the spinor, we find the following correspondence between the four types of order parameters and the electron densities (the spin index is omitted):

$$
\begin{aligned}
\mu & \rightarrow\left\langle\Psi^{\dagger} \Psi\right\rangle=n_{K_{+} A}+n_{K_{-} A}+n_{K_{+} B}+n_{K_{-} B}, \\
\tilde{\mu} & \rightarrow\left\langle\Psi^{\dagger} \gamma^{5} \Psi\right\rangle=n_{K_{+} A}-n_{K_{-} A}+n_{K_{+} B}-n_{K_{-} B}, \\
\Delta & \rightarrow\left\langle\bar{\Psi} \gamma^{3} \gamma^{5} \Psi\right\rangle=n_{K_{+} A}-n_{K_{-} A}-n_{K_{+} B}+n_{K_{-} B}, \\
\tilde{\Delta} & \rightarrow\left\langle\bar{\Psi} \gamma^{3} \Psi\right\rangle=n_{K_{+} A}+n_{K_{-} A}-n_{K_{+} B}-n_{K_{-} B},
\end{aligned}
$$

where the $n_{K_{+} A}, n_{K_{-} A}, n_{K_{+} B}$ and $n_{K_{-} B}$ are the densities of quasiparticles at specified valleys and sublattices. The QHF order parameters associated with $\mu$ and $\tilde{\mu}$ are the total density of electrons with a given spin and the density imbalance between the two valleys, respectively. Note that the $\mathrm{MC}$ order parameter related to the conventional Dirac mass $\tilde{\Delta}$ describes the density imbalance between the $A$ and $B$ sublattices (i.e., a charge density wave as interpreted in Refs. 24, 25, 26, 27, 29). The value of the singlet Dirac mass $\Delta$ [see Eq. (7)] controls a mixed density imbalance at the two valleys and the two sublattices.

In terms of symmetry, these order parameters can be divided in two groups. The order parameters $\left\langle\Psi^{\dagger} P_{s} \Psi\right\rangle$ and $\left\langle\bar{\Psi} \gamma^{3} \gamma^{5} P_{s} \Psi\right\rangle$ (related to $\mu_{s}$ and $\Delta_{s}$ ) with nonequal values for $s= \pm$ break the approximate $U(4)$ symmetry just like the Zeeman term, and they are singlets under the non-abelian subgroups $S U(2)_{s} \subset U(2)_{s}$ with the generators $P_{s} \otimes\left(-i \gamma^{3} / 2, \gamma^{3} \gamma^{5} / 2, \gamma^{5} / 2\right)$. Since these singlet order parameters break no exact symmetries of the action, they are not the order parameters in the strict sense. Yet, because of a relative smallness of the "bare" Zeeman energy $Z$ and a significant dynamical part in $\mu_{s}$ and $\Delta_{s}$, it is appropriate to talk about approximate spontaneous symmetry breaking. In the model in Refs. 31,32, these two order parameters coexist and play a crucial role in the solution corresponding to the $\nu=0$ plateau.

The order parameters of the other type, $\left\langle\Psi^{\dagger} \gamma^{5} P_{s} \Psi\right\rangle$ and $\left\langle\bar{\Psi} \gamma^{3} P_{s} \Psi\right\rangle$, (associated with $\tilde{\mu}_{s}$ and $\tilde{\Delta}_{s}$ ) are triplets under $S U(2)_{s} \subset U(2)_{s}$. Each of these two order parameters describes spontaneous $S U(2)_{s}$ symmetry breaking down to $U(1)_{s}$ with the generator $P_{s} \otimes \gamma^{5} / 2$. In the model in Refs. 31, 32, these two order parameters coexist and play an important role in the solution corresponding to the $\nu= \pm 1$ plateaus (as well as to the plateaus $\nu= \pm 3$ and $\nu= \pm 5$, which have not been observed yet).

\section{LANDAU LEVELS AND EDGE STATES}

In accordance with the discussion in the previous section, we assume that both ferromagnetic and mass type gaps coexist in general. Our goal is to find the spectrum of edge states in such a theory.

The structure of the inverse quasiparticle propagator in Eq. (3) implies that in the most general case the quadratic part of the effective Hamiltonian for quasiparticles of spin $s$ takes the following form in the first quantized theory:

$$
\hat{H}_{s}=\hat{H}_{0}-\mu_{s}-\tilde{\mu}_{s} \gamma^{5}-\Delta_{s} \gamma^{0} \gamma^{3} \gamma^{5}+\tilde{\Delta}_{s} \gamma^{0} \gamma^{3}
$$

The free part of the Hamiltonian reads

$$
\hat{H}_{0}=v_{F}\left(\alpha_{1} \pi_{x}+\alpha_{2} \pi_{y}\right)
$$

where $v_{F} \simeq 10^{6} \mathrm{~m} / \mathrm{s}$ is the Fermi velocity. By definition, $\alpha_{i}=\gamma^{0} \gamma^{i}$ and the canonical momentum is $\pi_{i}=-i \hbar \partial_{i}+e A_{i} / c$. Here the vector potential is taken in the Landau gauge: $A_{x}=-B y$ and $A_{y}=0$, where $B$ is the magnitude of a constant magnetic field orthogonal to the $x y$ plane of graphene.

The parameters $\mu_{s}, \tilde{\mu}_{s}, \Delta_{s}$ and $\tilde{\Delta}_{s}$ are determined from the gap equation. In particular, in the model in Refs. 31,32 , the $S 1$ solution (in the nomenclature of Ref. 32) near the Dirac neutral point, corresponding to the $\nu=0$ plateau, has the following form:

$$
\tilde{\Delta}_{ \pm}=\tilde{\mu}_{ \pm}=0, \mu_{ \pm}=\mp Z \mp A, \Delta_{ \pm}= \pm M
$$

where for the values of magnetic fields $B \lesssim 45 \mathrm{~T}$ utilized in the experiments in Refs. 13, 14, 15, 16, the dynamical parameters $A, M$ are considerably larger than $Z$, and $M>A$. The value of the spin gap in this solution is $\Delta E=$ $2 M+2(Z+A)$. Note that it is essentially larger than the spin gap $\Delta E=2(A+Z)$ in the QHF scenario. ${ }^{23}$ While the problem of calculating the values of $\mu_{s}, \tilde{\mu}_{s}, \Delta_{s}$ and $\tilde{\Delta}_{s}$ is not addressed in this study, it should be clear that in an actual device they are determined by (i) the strength of the magnetic field, (ii) the temperature, and (iii) other sample-specific parameters (e.g., the mobility of carriers, the size and geometry, the type of the substrate etc.). In practice, we analyze the spectrum of edge states in the model described by the model Hamiltonian in Eq. (9). 
In general, in a finite geometry case, the magnitude of the exchange and Hartree interactions which determine the values of the parameters $\Delta_{s}, \tilde{\Delta}_{s}, \mu_{s}$, and $\tilde{\mu}_{s}$ is likely to vary with the distance from the edge and should be calculated in a self-consistent way. The present study of the edge states is done assuming uniform gaps and uniform chemical potentials, and so it captures qualitative, but probably not quantitative aspects of the edge-state physics (see also a related discussion in the end of Sec. VI).

When written in components, the Dirac equation corresponding to the Hamiltonian (9) takes the following form:

$$
\begin{gathered}
\left(\begin{array}{cc}
E+\mu^{(+)}+\Delta^{(-)} & \hbar v_{F}\left(i D_{x}+D_{y}\right) \\
\hbar v_{F}\left(i D_{x}-D_{y}\right) & E+\mu^{(+)}-\Delta^{(-)}
\end{array}\right)\left(\begin{array}{l}
\psi_{K_{+} A} \\
\psi_{K_{+} B}
\end{array}\right)=0, \\
\left(\begin{array}{cc}
E+\mu^{(-)}+\Delta^{(+)} & -\hbar v_{F}\left(i D_{x}+D_{y}\right) \\
-\hbar v_{F}\left(i D_{x}-D_{y}\right) & E+\mu^{(-)}-\Delta^{(+)}
\end{array}\right)\left(\begin{array}{c}
\psi_{K_{-} B} \\
\psi_{K_{-} A}
\end{array}\right)=0 .
\end{gathered}
$$

Here the covariant derivative $D_{i}=\partial_{i}+(i e / \hbar c) A_{i}$ and the shorthand notations $\mu^{( \pm)} \equiv \mu \pm \tilde{\mu}$ and $\Delta( \pm) \equiv \Delta \pm \tilde{\Delta}$ were introduced (the spin index $s$ was omitted). In each of the two sets of equations, the $B$-components of the wave function can be eliminated,

$$
\begin{aligned}
\psi_{K_{+} B} & =\frac{\hbar v_{F}\left(-i D_{x}+D_{y}\right) \psi_{K_{+} A}}{E+\mu^{(+)}-\Delta^{(-)}}, \\
\psi_{K_{-} B} & =\frac{\hbar v_{F}\left(i D_{x}+D_{y}\right) \psi_{K_{-}}}{E+\mu^{(-)}+\Delta^{(+)}} .
\end{aligned}
$$

By taking these into account, we derive the equations for the $A$-components,

$$
\begin{aligned}
& \left(-l^{2} D_{x}^{2}-l^{2} D_{y}^{2}+1\right) \psi_{K_{+} A}=2 \lambda_{+} \psi_{K_{+} A}, \\
& \left(-l^{2} D_{x}^{2}-l^{2} D_{y}^{2}-1\right) \psi_{K_{-} A}=2 \lambda_{-} \psi_{K_{-} A},
\end{aligned}
$$

where $\lambda_{ \pm}=\left[\left(E+\mu^{( \pm)}\right)^{2}-\left(\Delta^{(\mp)}\right)^{2}\right] / \epsilon_{0}^{2}, l \equiv \sqrt{\hbar c /|e B|}$ is the magnetic length, and $\epsilon_{0} \equiv \sqrt{2 \hbar v_{F}^{2}|e B| / c}$ is the Landau energy scale. Note that $\lambda_{+}\left(\lambda_{-}\right)$is related to the $K_{+}\left(K_{-}\right)$valley.

In the Landau gauge $\mathbf{A}=(-B y, 0)$, the wave functions are plane waves in the $x$-direction. Thus, we write

$$
\begin{aligned}
& \psi_{K_{+} A}(\mathbf{r}, k)=\frac{e^{i k x}}{\sqrt{2 \pi l}} u_{+}(y, k), \quad \psi_{K_{+} B}(\mathbf{r}, k)=\frac{e^{i k x}}{\sqrt{2 \pi l}} v_{+}(y, k), \\
& \psi_{K_{-} A}(\mathbf{r}, k)=\frac{e^{i k x}}{\sqrt{2 \pi l}} u_{-}(y, k), \quad \psi_{K_{-} B}(\mathbf{r}, k)=\frac{e^{i k x}}{\sqrt{2 \pi l}} v_{-}(y, k) .
\end{aligned}
$$

The envelope functions $u_{ \pm}(y, k)$ and $v_{ \pm}(y, k)$ depend only on a single combination of the variables, $\xi=y / l-k l$, and satisfy the following equations:

$$
\begin{aligned}
& \left(\partial_{\xi}^{2}-\xi^{2} \mp 1+2 \lambda_{ \pm}\right) u_{ \pm}(\xi)=0, \\
& v_{ \pm}(\xi)=\frac{\epsilon_{0}\left(\partial_{\xi} \mp \xi\right) u_{ \pm}(\xi)}{\sqrt{2}\left(E+\mu^{( \pm)} \mp \Delta^{(\mp)}\right)} .
\end{aligned}
$$

Note that the wavevector $k$ determines the center of the electron orbital along the $y$-direction, $y_{k}=k l^{2}$. Then, as we shall see below, for a system with a ribbon geometry, e.g., $0 \leq y \leq W$, the condition of finite energy will be satisfied only for eigenstates with wavevectors $k$ in a finite range, $0 \lesssim k \lesssim W / l^{2}$. This is known as the position-wavevector duality in the Landau gauge. (Note that the maximum value of the wavevector $k$, measuring the displacement from either $K_{+}$or $K_{-}$point, is limited by the boundaries of the first Brillouin zone. However, this fact is not explicit in the low-energy theory.)

The general solution to Eqs. (20) and (21) is given in terms of the parabolic cylinder (Weber) functions $U(a, z)$ and $V(a, z), \underline{44}$

$$
\begin{aligned}
& u_{+}(\xi)=C_{1} \frac{E+\mu^{(+)}-\Delta^{(-)}}{\epsilon_{0}} U\left(\frac{1-2 \lambda_{+}}{2}, \sqrt{2} \xi\right)+C_{2} V\left(\frac{1-2 \lambda_{+}}{2}, \sqrt{2} \xi\right), \\
& v_{+}(\xi)=-C_{1} U\left(-\frac{1+2 \lambda_{+}}{2}, \sqrt{2} \xi\right)-C_{2} \frac{E+\mu^{(+)}+\Delta^{(-)}}{\epsilon_{0}} V\left(-\frac{1+2 \lambda_{+}}{2}, \sqrt{2} \xi\right), \\
& u_{-}(\xi)=C_{3} U\left(-\frac{1+2 \lambda_{-}}{2}, \sqrt{2} \xi\right)+C_{4} \frac{E+\mu^{(-)}+\Delta^{(+)}}{\epsilon_{0}} V\left(-\frac{1+2 \lambda_{-}}{2}, \sqrt{2} \xi\right) \\
& v_{-}(\xi)=C_{3} \frac{E+\mu^{(-)}-\Delta^{(+)}}{\epsilon_{0}} U\left(\frac{1-2 \lambda_{-}}{2}, \sqrt{2} \xi\right)+C_{4} V\left(\frac{1-2 \lambda_{-}}{2}, \sqrt{2} \xi\right) .
\end{aligned}
$$



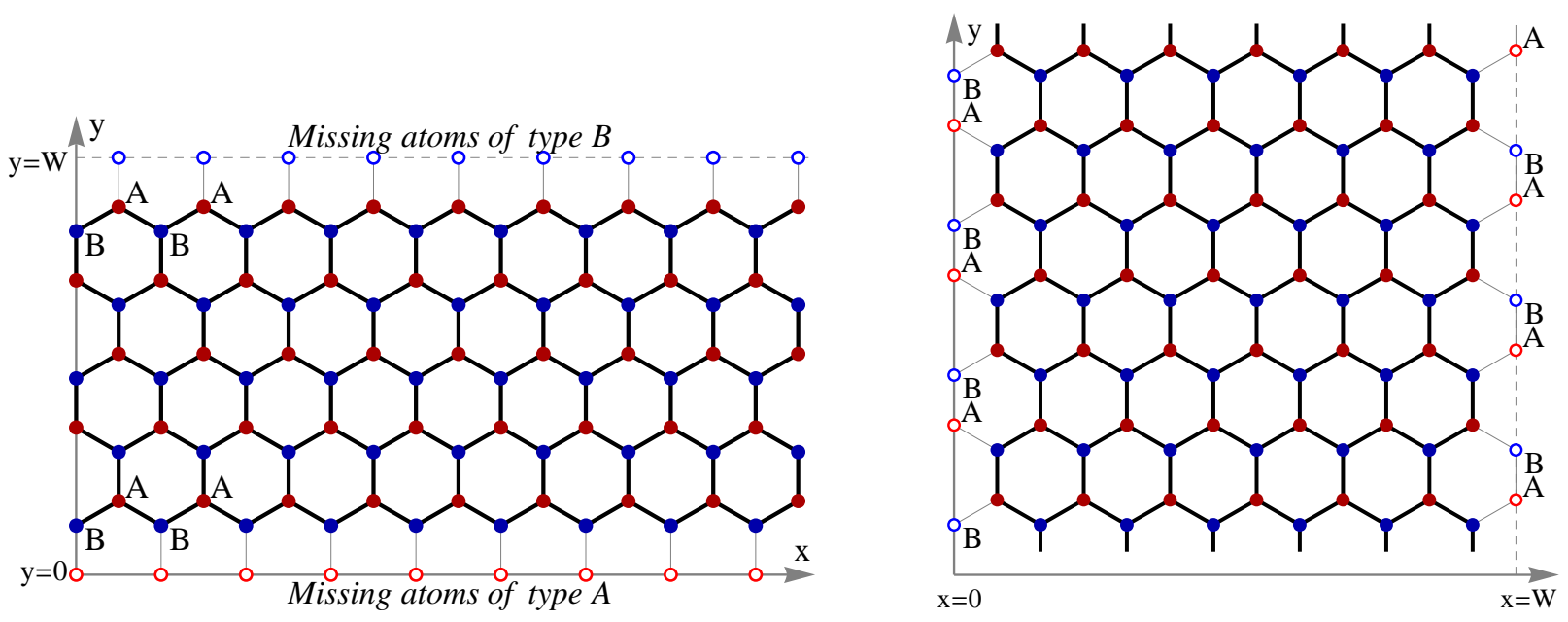

FIG. 1: (Color online) The lattice structure of a finite width graphene ribbon with zigzag (left panel) and armchair (right panel) edges.

Note the following relations with the parabolic cylinder functions $D_{\nu}(z)$ :

$$
\begin{aligned}
U(a, z) & =D_{-a-1 / 2}(z), \\
V(a, z) & =\frac{\Gamma(a+1 / 2)}{\pi}\left[\sin (\pi a) D_{-a-1 / 2}(z)+D_{-a-1 / 2}(-z)\right] .
\end{aligned}
$$

In an infinite system, the normalizable wave functions contain only the parabolic cylinder $U$-functions which are bound at $z \rightarrow \pm \infty$ provided $a=-n-1 / 2$ and $n$ is a non-negative integer. In fact, the following relation is valid: $U(-n-1 / 2, z)=2^{-n / 2} e^{-z^{2} / 4} H_{n}(z / \sqrt{2})$, where $H_{n}(z)$ are the Hermite polynomials. In this case, the spectrum is determined by $\lambda_{ \pm}^{(b u l k)}=n$ with $n=0,1,2, \ldots$

In the case of a graphene ribbon of a finite width in the $y$-direction, $0 \leq y \leq W$, and with two zigzag edges parallel to the $x$-direction, the $A$ - and $B$-components of wave functions should vanish on the opposite edges $\frac{23,38}{}$, i.e.,

$$
\begin{aligned}
& y=0: \quad u_{+}(-k l)=u_{-}(-k l)=0 \\
& y=W: \quad v_{+}(W / l-k l)=v_{-}(W / l-k l)=0
\end{aligned}
$$

see Fig. 1 In principle, by satisfying these equations and using the wave function normalization conditions, we can determine all four integration constants in Eqs. (22)-(25). For our purposes here, however, it suffices to determine the conditions when non-trivial, normalizable solutions exist. These will provide the dispersion spectra of all modes in a ribbon of graphene. The corresponding numerical analysis is presented in Sec. IV]

In the case of a graphene ribbon with armchair edges parallel to the $y$-direction, it is convenient to choose a different Landau gauge with $\left(A_{x}, A_{y}\right)=(0, B x)$. Accordingly, the solutions are translation invariant along the $y$-direction,

$$
\begin{aligned}
\psi_{A K}(\mathbf{r}, k)=\frac{1}{\sqrt{2 \pi l}} e^{i k y} u_{+}(x, k), & \psi_{B K}(\mathbf{r}, k)=\frac{1}{\sqrt{2 \pi l}} e^{i k y} v_{+}(x, k), \\
\psi_{A K_{-}}(\mathbf{r}, k)=\frac{1}{\sqrt{2 \pi l}} e^{i k y} u_{-}(x, k), & \psi_{B K_{-}}(\mathbf{r}, k)=\frac{1}{\sqrt{2 \pi l}} e^{i k y} v_{-}(x, k) .
\end{aligned}
$$

Then, the corresponding differential equations for functions $u_{ \pm}(x, k)$, which are rewritten in terms of the dimensionless variable $\xi=x / l+k l$, coincide with Eq. (20). The expressions for the eliminated components $v_{ \pm}(\xi)$, however, slightly differ from Eq. (21), and are given by

$$
v_{ \pm}(\xi)=\mp i \frac{\epsilon_{0}\left(\partial_{\xi} \mp \xi\right) u_{ \pm}(\xi)}{\sqrt{2}\left(E+\mu^{( \pm)} \mp \Delta^{(\mp)}\right)} .
$$


The general solutions for the $u_{ \pm}(\xi)$ functions have the same form as in Eqs. (22) and (24),

$$
\begin{aligned}
& u_{+}(\xi)=C_{1} \frac{E+\mu^{(+)}-\Delta^{(-)}}{\epsilon_{0}} U\left(\frac{1-2 \lambda_{+}}{2}, \sqrt{2} \xi\right)+C_{2} V\left(\frac{1-2 \lambda_{+}}{2}, \sqrt{2} \xi\right), \\
& u_{-}(\xi)=C_{3} U\left(-\frac{1+2 \lambda_{-}}{2}, \sqrt{2} \xi\right)+C_{4} \frac{E+\mu^{(-)}+\Delta^{(+)}}{\epsilon_{0}} V\left(-\frac{1+2 \lambda_{-}}{2}, \sqrt{2} \xi\right),
\end{aligned}
$$

but with $\xi=x / l+k l$. By using the relations in Eq. (32), we also obtain the explicit expression for $v_{ \pm}(\xi)$ functions,

$$
\begin{aligned}
& v_{+}(\xi)=i C_{1} U\left(-\frac{1+2 \lambda_{+}}{2}, \sqrt{2} \xi\right)+i C_{2} \frac{E+\mu^{(+)}+\Delta^{(-)}}{\epsilon_{0}} V\left(-\frac{1+2 \lambda_{+}}{2}, \sqrt{2} \xi\right) \\
& v_{-}(\xi)=i C_{3} \frac{E+\mu^{(-)}-\Delta^{(+)}}{\epsilon_{0}} U\left(\frac{1-2 \lambda_{-}}{2}, \sqrt{2} \xi\right)+i C_{4} V\left(\frac{1-2 \lambda_{-}}{2}, \sqrt{2} \xi\right) .
\end{aligned}
$$

Since the armchair edges have lattice sites of both $A$ and $B$ types, the wave function should vanish both at the $x=0$ and $x=W$ lines $, 23,36,38$

$$
\begin{array}{rll}
x=0: & u_{+}(k l)+u_{-}(k l)=0, & v_{+}(k l)+v_{-}(k l)=0, \\
x=W: & u_{+}(W / l+k l)+u_{-}(W / l+k l)=0, & v_{+}(W / l+k l)+v_{-}(W / l+k l)=0 .
\end{array}
$$

It is important to notice that the armchair boundary conditions mix the chiralities associated with the $K_{+}$and $K_{-}$ valleys, which makes the analysis more involved than in the case of zigzag edges. The details are given in Sec. $\mathrm{V}$.

\section{NUMERICAL RESULTS IN THE CASE OF ZIGZAG EDGES.}

Let us start from the boundary conditions at $K_{+}$valley, see (28) and (29). They take the following explicit form:

$$
\begin{aligned}
& C_{1} \frac{E+\mu^{(+)}-\Delta^{(-)}}{\epsilon_{0}} U\left(\frac{1-2 \lambda_{+}}{2},-\sqrt{2} k l\right)=-C_{2} V\left(\frac{1-2 \lambda_{+}}{2},-\sqrt{2} k l\right), \\
& C_{1} U\left(-\frac{1+2 \lambda_{+}}{2}, \sqrt{2}\left(k_{0}-k\right) l\right)=-C_{2} \frac{E+\mu^{(+)}+\Delta^{(-)}}{\epsilon_{0}} V\left(-\frac{1+2 \lambda_{+}}{2}, \sqrt{2}\left(k_{0}-k\right) l\right),
\end{aligned}
$$

where $k_{0} \equiv W / l^{2}$ is determined by the width of the ribbon. A nontrivial solution to this set of equations exists when

$$
\lambda_{+} U\left(\frac{1-2 \lambda_{+}}{2},-\sqrt{2} k l\right) V\left(-\frac{1+2 \lambda_{+}}{2}, \sqrt{2}\left(k_{0}-k\right) l\right)-U\left(-\frac{1+2 \lambda_{+}}{2}, \sqrt{2}\left(k_{0}-k\right) l\right) V\left(\frac{1-2 \lambda_{+}}{2},-\sqrt{2} k l\right)=0 .
$$

A similar condition is derived at $K_{-}$valley,

$\lambda_{-} U\left(\frac{1-2 \lambda_{-}}{2}, \sqrt{2}\left(k_{0}-k\right) l\right) V\left(-\frac{1+2 \lambda_{-}}{2},-\sqrt{2} k l\right)-U\left(-\frac{1+2 \lambda_{-}}{2},-\sqrt{2} k l\right) V\left(\frac{1-2 \lambda_{-}}{2}, \sqrt{2}\left(k_{0}-k\right) l\right)=0$.

By solving Eqs. (411) and (42) numerically, we determine the dependence of dimensionless energy parameters $\lambda_{+}$and $\lambda_{-}$on the wavevector $k$. The results for two different widths of graphene ribbons, $W=5 l$ and $W=10 l$, are shown in Fig. 2].

When the width of the ribbon is less than about 3 or 4 times the magnetic length $l \simeq 257 \AA / \sqrt{B[\mathrm{~T}]}$, we find that the spectra have little overlap with the usual bulk spectra, i.e., $\lambda_{ \pm}^{(b u l k)}=n$ where $n$ is a non-negative integer. Additionally, the separation between the nearest levels quickly increases with decreasing $W$.

For the case $W=5 l$, shown in the left panel of Fig. 2] only the lowest level may have a hint at the middle plateau developing. However, when the ribbon's width is larger than about 6 or 7 times the magnetic length, nearly flat plateaus are already distinguishable in the lowest levels around the central wavevector $\frac{1}{2} k_{0}$. We also find that the lower the level, the wider the plateau formed.

Let us also emphasize the following special feature of the spectrum in a graphene ribbon with zigzag boundaries. As we see from Fig. 2. for $\lambda_{+} \simeq 0$ (actually, $\left.E \simeq-\mu^{(+)}+\Delta^{(-)}\right)$and for $\lambda_{-} \simeq 0$ (actually, $E \simeq-\mu^{(-)}+\Delta^{(+)}$), dispersionless 

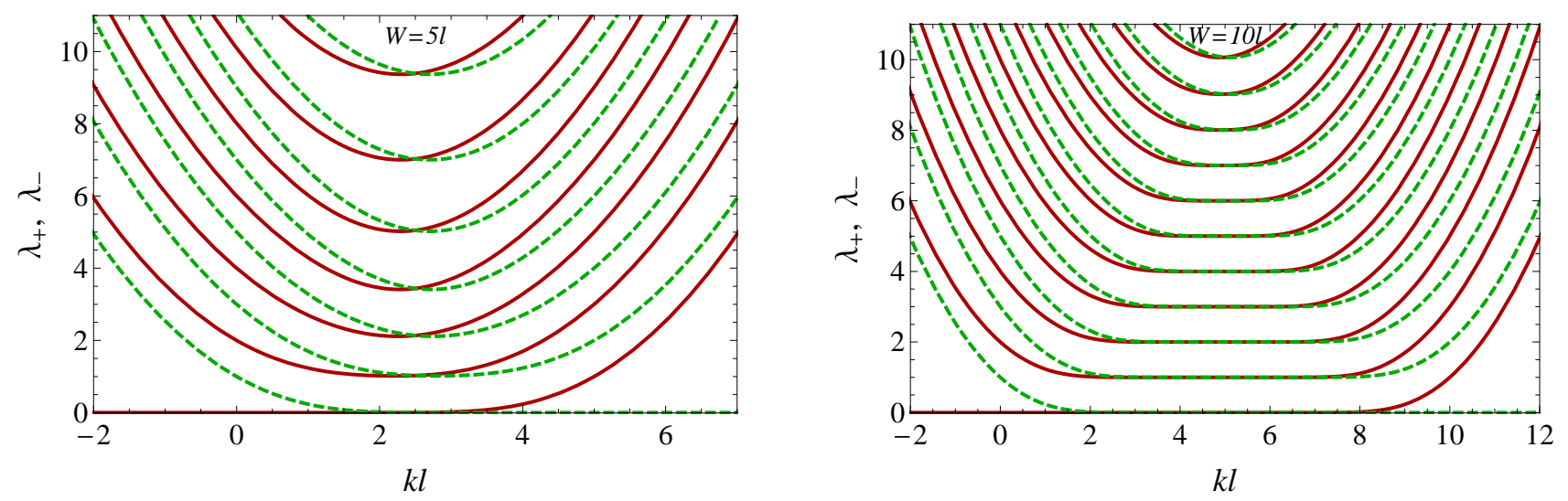

FIG. 2: (Color online) Numerical results for the dimensionless parameters $\lambda_{+}$(solid lines) and $\lambda_{-}$(dashed lines) in the case of ribbons with zigzag edges. The ribbons' widths are $W=5 l$ (left panel) and $W=10 l$ (right panel).

surface solutions $\underline{23,38}$ exist at both valleys (note that such solutions exist also in the case with no magnetic field $\stackrel{35,39}{ }$ ). These solutions are bound to the $k \simeq 0$ and $k \simeq k_{0}$ edges for the $K_{+}$and $K_{-}$valleys, respectively. It is noticeable that unlike the case of a half-plane, $\stackrel{42}{\stackrel{4}{2}}$ they cease to be dispersionless at the opposite edges, i.e., at $k \simeq k_{0}(k \simeq 0)$ for the $K_{+}\left(K_{-}\right)$valley, respectively.

Now, by restoring the spin index, we assemble the complete spectrum of a graphene ribbon described by Hamiltonian (9) with dynamical order parameters proposed in Refs. 31,32,

$$
\begin{aligned}
& E_{s K_{+}}^{( \pm)}(n, k)=-\mu_{s}^{(+)} \pm \sqrt{\lambda_{+}(n, k) \epsilon_{0}^{2}+\left(\Delta_{s}^{(-)}\right)^{2}}, \\
& E_{s K_{-}}^{( \pm)}(n, k)=-\mu_{s}^{(-)} \pm \sqrt{\lambda_{-}(n, k) \epsilon_{0}^{2}+\left(\Delta_{s}^{(+)}\right)^{2}} .
\end{aligned}
$$

Notice that there exist eight sublevels that correspond to the lowest Landau level. Only half of these correspond to the bulk states, i.e., those which remain normalizable on an infinite graphene plane. The other half have wave functions localized only at the edges. In fact, using the properties of the parabolic cylinder functions, one can show that in the coordinate space, the wave functions of the additional branches of solutions (i.e., those which disappear on an infinite plane) are localized near either $y=0$ or $y=W$ edges of the ribbon for all values of the quantum number $k$ (the non-bulk states). In other words, the earlier mentioned position-wavevector duality can be used only for the description of the branches of the bulk states.

By making use of the numerical results for $\lambda_{+}$and $\lambda_{-}$, we can plot the actual energy spectra in the system. For the ribbons of widths $W=5 l$ and $W=10 l$, these are presented in Figs. 3 and 4 . Here the choice of the order parameters resembles those of the solution in Eq. (11) corresponding to the Dirac neutral point, i.e., the $\nu=0$ plateau $\stackrel{31,32}{=}$ However, in order to lift the degeneracy of all sublevels, we also added small non-zero values for the triplet chemical potentials $\tilde{\mu}_{ \pm}$.

By considering different relative strengths of ferromagnetic and mass gaps in Figs. 3 and 4 , we see that there exist gapless edge states (whose energy vanishes at certain values of $k$ ) only when the ferromagnetic gap dominates over the mass gap, i.e., $\left|\mu_{s}^{( \pm)}\right|>\left|\Delta_{s}^{(\mp)}\right|$. (The values of wavevectors that give gapless modes are marked by the dots in the spectra in Fig. 3]) From Fig. 2, we can see that $\lambda_{ \pm}(0, k)$ is nonnegative and approaches zero at certain values of the wavevector. This feature together with dispersion relations (43) and (44) makes it clear that the necessary and sufficient condition for the existence of gapless modes is that at least one of the inequalities $\left|\mu_{s}^{(-)}\right|>\left|\Delta_{s}^{(+)}\right|$, $\left|\mu_{s}^{(+)}\right|>\left|\Delta_{s}^{(-)}\right|$is satisfied for at least one spin choice.

So far, we have considered only the case with nonzero singlet Dirac masses. However, as is clear from Eqs. (43) and (44), the results for nonzero triplet Dirac masses will look exactly the same as in Figs. 3 and 4 . At first sight, this might appear to be surprising because the symmetry properties of the two types of the masses are different: while the triplet masses break the $S U(2)_{s}$ valley symmetries, the singlet masses do not. Let us turn to the discussion of this point. First of all, the corresponding symmetry is exact only on an infinite plane. As to a finite width ribbon, it is explicitly broken by the boundary conditions there, as seen from the comparison of Eq. (41) for $K_{+}$valley and Eq. (42) for $K_{-}$ valley. This is also obvious from the solutions for $\lambda_{ \pm}$in Fig. 2, On the other hand, although one might expect that the symmetry arguments are to be approximately applicable to the states with intermediate values of the wavevector 

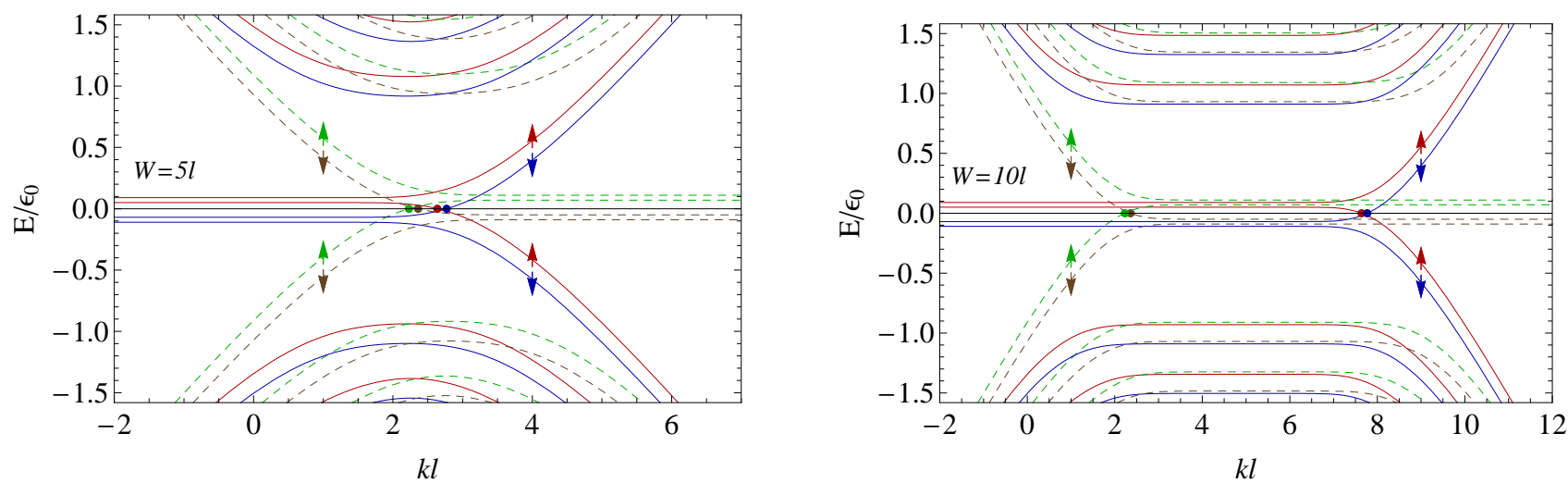

FIG. 3: (Color online) Numerical results for the low-energy spectra for ribbons with zigzag edges. The ribbons' widths are $W=5 l$ (left panel) and $W=10 l$ (right panel). The ferromagnetic gaps and dynamical masses are as follows: $\mu_{ \pm}=\mp 0.08 \epsilon_{0}$, $\tilde{\mu}_{ \pm}=0.01 \epsilon_{0}, \Delta_{ \pm}= \pm 0.02 \epsilon_{0}, \tilde{\Delta}_{ \pm}=0$. The ferromagnetic gap dominates over the mass gap, insuring the presence of gapless edge states (marked by dots). The electron spins of the lowest energy sublevels are marked by arrows. The spectra around the $K_{+}\left(K_{-}\right)$point are shown by solid (dashed) lines.
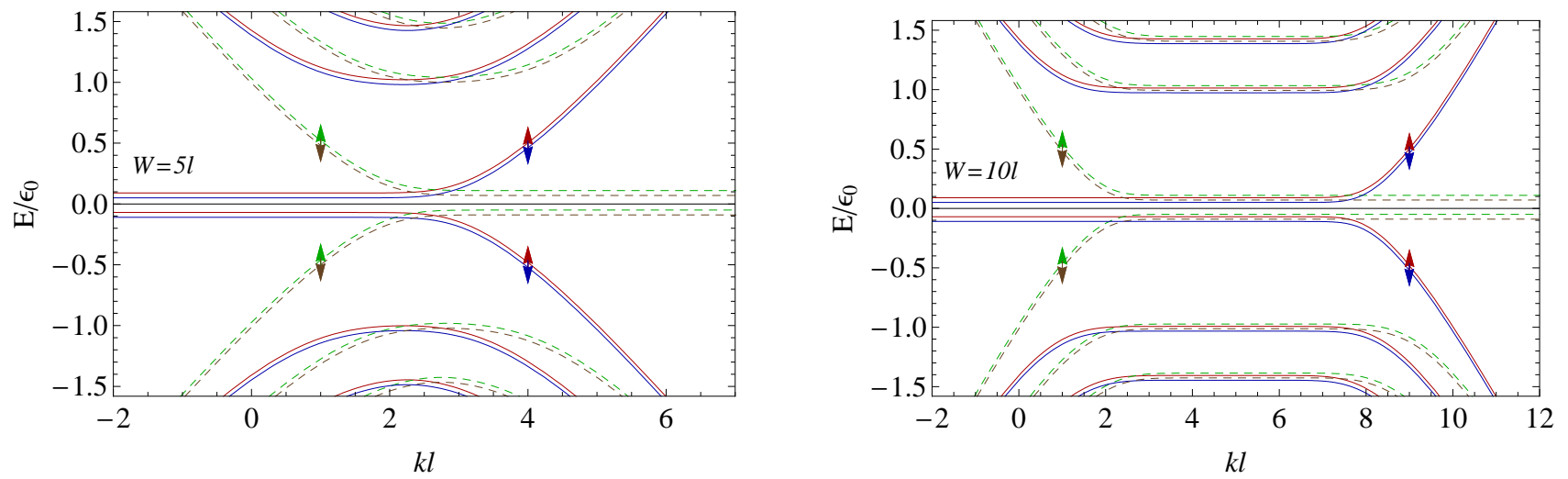

FIG. 4: (Color online) Same as Fig. 3 but with the ferromagnetic gaps and dynamical masses being as follows: $\mu_{ \pm}=\mp 0.02 \epsilon_{0}$, $\tilde{\mu}_{ \pm}=0.01 \epsilon_{0}, \Delta_{ \pm}= \pm 0.08 \epsilon_{0}, \tilde{\Delta}_{ \pm}=0$. The mass gap dominates over the ferromagnetic gap, insuring the absence of gapless edge states.

$k$ in a bulk of a ribbon, both these types of the Dirac masses still lead to the same spectra there. This puzzle is resolved as follows. As was already emphasized above, one should distinguish between the bulk states and non-bulk ones on a ribbon. The dispersion relations in the ribbon bulk for the former approximately coincide with those of the states on an infinite plane. In the case of the LLL, they are $E_{s K_{+}}=-\mu_{s}^{(+)}+\Delta_{s}^{(-)}$and $E_{s K_{-}}=-\mu_{s}^{(-)}+\Delta_{s}^{(+)} \underline{\underline{31}, 32}$ The point is that on the ribbon, the asymmetry of this spectrum for $\tilde{\Delta}_{s} \neq 0$ is washed away by the inclusion of four additional non-bulk branches, which are localized exclusively on the edges and whose energies are approximately given by similar expressions but with the opposite signs in front of $\Delta_{s}^{( \pm)}$. As for higher LLs, it is known that their energies do not depend on the type of Dirac masses even on an infinite plane, provided only a mass of one type is present $\underline{\underline{31,32}}$ Thus, on a ribbon, the sublevel structure of the LLL becomes similar to that of higher LLs.

It is instructive to compare the properties of the solutions on a finite width ribbon with those on a half-plane. $\stackrel{42}{*}$ First of all, instead of the eight sublevels of the LLL on a ribbon, there are only six ones on a half-plane. The reason of that is of course connected with the fact that each edge adds two additional sublevels connected with two orientations of spin. Secondly, unlike the case of a ribbon, there are LLL sublevels which are dispersionless for all values of $k$ on a half-plane. This feature can be also easily understood. As the width $W$ of a ribbon goes to infinity, the edge $k=k_{0}$ disappears and the solutions which are bound to the edge $k=0$ become dispersionless for all values $k>0$.

In relation to the dispersionless modes, we would like to add the following comment. Because of level crossing, there is an ambiguity in the definition of a single branch of solutions in the LLL. By mixing together the two branches 
associated with different valleys, one can construct a new branch that is approximately dispersionless for all values of

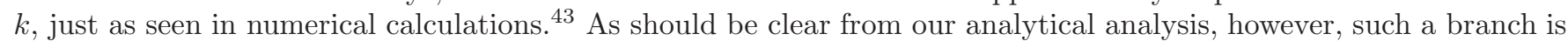
only approximately dispersionless for intermediate values of the wavevector $k$.

\section{NUMERICAL RESULTS IN THE CASE OF ARMCHAIR EDGES.}

Let us now consider the case of the armchair edges. Since the armchair boundary conditions (37) and (38) mix the chiralities associated with the $K_{+}$and $K_{-}$valleys, this case is essentially more complicated for both the analytic and numerical analyses on a ribbon.

The explicit form of the boundary conditions (37) and (38) reads:

$$
\begin{gathered}
C_{1} \frac{E+\mu^{(+)}-\Delta^{(-)}}{\epsilon_{0}} U\left(\frac{1-2 \lambda_{+}}{2}, \sqrt{2} k l\right)+C_{2} V\left(\frac{1-2 \lambda_{+}}{2}, \sqrt{2} k l\right)+ \\
+C_{3} U\left(-\frac{1+2 \lambda_{-}}{2}, \sqrt{2} k l\right)+C_{4} \frac{E+\mu^{(-)}+\Delta^{(+)}}{\epsilon_{0}} V\left(-\frac{1+2 \lambda_{-}}{2}, \sqrt{2} k l\right)=0, \\
C_{1} U\left(-\frac{1+2 \lambda_{+}}{2}, \sqrt{2} k l\right)+C_{2} \frac{E+\mu^{(+)}+\Delta^{(-)}}{\epsilon_{0}} V\left(-\frac{1+2 \lambda_{+}}{2}, \sqrt{2} k l\right)+ \\
+C_{3} \frac{E+\mu^{(-)}-\Delta^{(+)}}{\epsilon_{0}} U\left(\frac{1-2 \lambda_{-}}{2}, \sqrt{2} k l\right)+C_{4} V\left(\frac{1-2 \lambda_{-}}{2}, \sqrt{2} k l\right)=0, \\
C_{1} \frac{E+\mu^{(+)}-\Delta^{(-)}}{\epsilon_{0}} U\left(\frac{1-2 \lambda_{+}}{2}, \sqrt{2}\left(k-k_{0}\right) l\right)+C_{2} V\left(\frac{1-2 \lambda_{+}}{2}, \sqrt{2}\left(k-k_{0}\right) l\right)+ \\
+C_{3} U\left(-\frac{1+2 \lambda_{-}}{2}, \sqrt{2}\left(k-k_{0}\right) l\right)+C_{4} \frac{E+\mu^{(-)}+\Delta^{(+)}}{\epsilon_{0}} V\left(-\frac{1+2 \lambda_{-}}{2}, \sqrt{2}\left(k-k_{0}\right) l\right)=0, \\
C_{1} U\left(-\frac{1+2 \lambda_{+}}{2}, \sqrt{2}\left(k-k_{0}\right) l\right)+C_{2} \frac{E+\mu^{(+)}+\Delta^{(-)}}{\epsilon_{0}} V\left(-\frac{1+2 \lambda_{+}}{2}, \sqrt{2}\left(k-k_{0}\right) l\right)+ \\
+C_{3} \frac{E+\mu^{(-)}-\Delta^{(+)}}{\epsilon_{0}} U\left(\frac{1-2 \lambda_{-}}{2}, \sqrt{2}\left(k-k_{0}\right) l\right)+C_{4} V\left(\frac{1-2 \lambda_{-}}{2}, \sqrt{2}\left(k-k_{0}\right) l\right)=0,
\end{gathered}
$$

where $k_{0}=-W / l^{2}$. It is a homogeneous system of four linear equations for four unknown constants $C_{i}, i=1,2,3,4$. The nontrivial solution exists when the determinant of a matrix made of the coefficients is zero. We numerically solve the corresponding equation to determine the spectrum of single-particle states in the graphene ribbon.

The cases with nonzero singlet $\left(\Delta_{s}\right)$ and triplet $\left(\tilde{\Delta}_{s}\right)$ Dirac masses are studied separately. Numerical results for singlet masses are shown in Fig. 5 . We find that gapless edge states always appear when there are only Zeemanlike (QHF) gap and singlet Dirac masses $\Delta_{s}$, irrespective of the actual relation between their values. We conclude, therefore, that for the case of a singlet Dirac mass, the condition for the existence of gapless modes is less constrained at the armchair edges than at the zigzag ones. In fact, this result should have been expected after recalling that the singlet Dirac masses do not break the global valley symmetry groups $S U(2)_{s} \subset U(2)_{s}$. These symmetries (one for spin up and the other for spin down) protect the double degeneracy of the Landau levels in the bulk. The property of such sublevels in the LLL is that they repel in opposite directions near the edges both in the absence of singlet Dirac masses and when they are present. The latter leads to gapless states. (Note that in this argument we implicitly use the fact that the energy separation of the sublevels may become arbitrarily large. This is not true on a lattice. However, even on the lattice the energy separation may become much larger than the dynamical scale of the mass, suggesting that the conclusion is still valid in that case.) In fact, the absence of gapless modes on a ribbon with zigzag edges and $\left|\Delta_{s}\right|>\left|\mu_{s}\right|$ is a special property, which is related to the dispersionless nature of the LLL modes at the edges with $k \simeq 0$ or $k \simeq k_{0}$.

Let us now turn to a triplet Dirac mass. We found that the analysis of the energy spectra can be considerably simplified in the case of nonzero triplet Dirac masses but vanishing $\tilde{\mu}_{s}$ and $\Delta_{s}$. The central observation is that in this case the determinant of the matrix corresponding to Eqs. (45)-(48) can be reduced to factorized form, and the spectral equation becomes

$$
f_{+}(k, \lambda) f_{-}(k, \lambda)=0,
$$



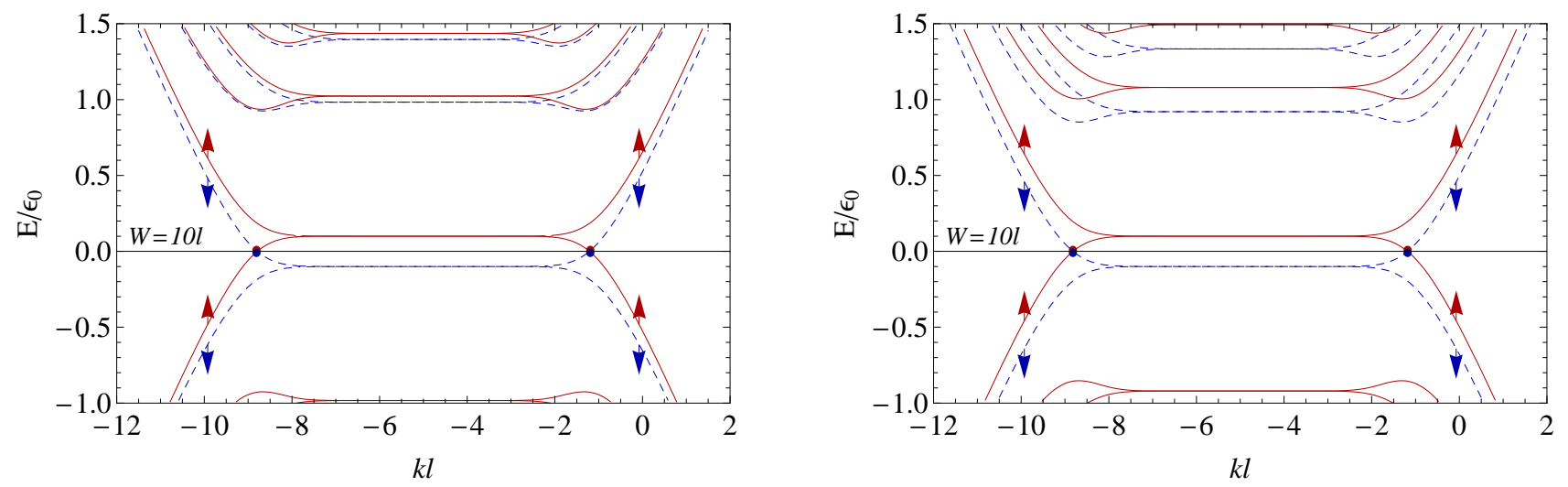

FIG. 5: (Color online) Numerical results for the low-energy spectra for a ribbon with armchair edges of width $W=10 l$ in the case of nonzero spin splitting and nonzero singlet masses. Nonzero dynamical parameters are as follows: $\mu_{ \pm}=\mp 0.02 \epsilon_{0}$, $\Delta_{ \pm}= \pm 0.08 \epsilon_{0}$ (left panel) and $\mu_{ \pm}=\mp 0.08 \epsilon_{0}, \Delta_{ \pm}= \pm 0.02 \epsilon_{0}$ (right panel). Gapless edge states (marked by dots) are present in both cases. The electron spins of the lowest energy sublevels are marked by arrows.

where, by definition, $\lambda=\left[(E+\mu)^{2}-\tilde{\Delta}^{2}\right] / \epsilon_{0}^{2}$ and

$$
\begin{aligned}
f_{ \pm}(k, \lambda) & =U\left(-\frac{1+2 \lambda}{2}, \sqrt{2}\left(k-k_{0}\right) l\right) V\left(\frac{1-2 \lambda}{2}, \sqrt{2} k l\right)-U\left(-\frac{1+2 \lambda}{2}, \sqrt{2} k l\right) V\left(\frac{1-2 \lambda}{2}, \sqrt{2}\left(k-k_{0}\right) l\right) \\
& +\lambda\left[U\left(\frac{1-2 \lambda}{2}, \sqrt{2}\left(k-k_{0}\right) l\right) V\left(-\frac{1+2 \lambda}{2}, \sqrt{2} k l\right)-U\left(\frac{1-2 \lambda}{2}, \sqrt{2} k l\right) V\left(-\frac{1+2 \lambda}{2}, \sqrt{2}\left(k-k_{0}\right) l\right)\right] \\
& \pm \sqrt{\lambda}\left[U\left(\frac{1-2 \lambda}{2}, \sqrt{2}\left(k-k_{0}\right) l\right) V\left(\frac{1-2 \lambda}{2}, \sqrt{2} k l\right)-U\left(\frac{1-2 \lambda}{2}, \sqrt{2} k l\right) V\left(\frac{1-2 \lambda}{2}, \sqrt{2}\left(k-k_{0}\right) l\right)\right. \\
& \left.+U\left(-\frac{1+2 \lambda}{2}, \sqrt{2}\left(k-k_{0}\right) l\right) V\left(-\frac{1+2 \lambda}{2}, \sqrt{2} k l\right)-U\left(-\frac{1+2 \lambda}{2}, \sqrt{2} k l\right) V\left(-\frac{1+2 \lambda}{2}, \sqrt{2}\left(k-k_{0}\right) l\right)\right] .
\end{aligned}
$$

Restoring the spin index again, the complete energy spectrum takes the form:

$$
E_{s n}(k)=-\mu_{s} \pm \sqrt{\lambda(k l, n) \epsilon_{0}^{2}+\tilde{\Delta}_{s}^{2}}
$$

where $n=0,1,2, \ldots$. Our numerical results for several lowest branches of $\lambda$ versus $k l$ are presented in Fig. 6. Note that $\lambda(k l, n) \simeq n$ at intermediate values of $k l$. This means that the low-energy spectrum of a finite width ribbon far from the edges should be approximately the same as the spectrum of graphene on an infinite plane.

The energy spectrum in the case of triplet Dirac masses are shown in Fig. 7 As seen from the figure, the presence of gapless modes in the spectrum sensitively depends on the relation between the values of the Zeeman-like (QHF) gaps and the triplet Dirac masses $\tilde{\Delta}_{s}$. Such modes exist only when the magnitude of the Dirac mass is less than the QHF gap. The physics underlying this result is clear. When $\left|\tilde{\Delta}_{s}\right|>\left|\mu_{s}\right|$, the valley splitting is large and there are two LLL branches of states with opposite signs of their energies for each direction of the spin [see Eq. (51) and the left panel in Fig. (7). As a result, repelling of these branches at the edges does not lead to the creation of gapless modes. On the other hand, in the case of $\left|\tilde{\Delta}_{s}\right|<\left|\mu_{s}\right|$, shown in the right panel of Fig. (7) [see also Eq. (51)], the valley splitting is small, the energies of the two branches have the same sign, and the process of their repelling at the edges inevitably creates gapless modes. In essence, this is the same condition as for zigzag edges in a system with triplet Dirac masses studied in the previous section,

$$
\left|\tilde{\Delta}_{s}\right|<\left|\mu_{s}\right|
$$

\section{DISCUSSION}

The main result in this paper is establishing the criterion for the existence of gapless modes among the edge states in a graphene ribbon with zigzag and armchair type edges. The method used in this paper combines analytic and 


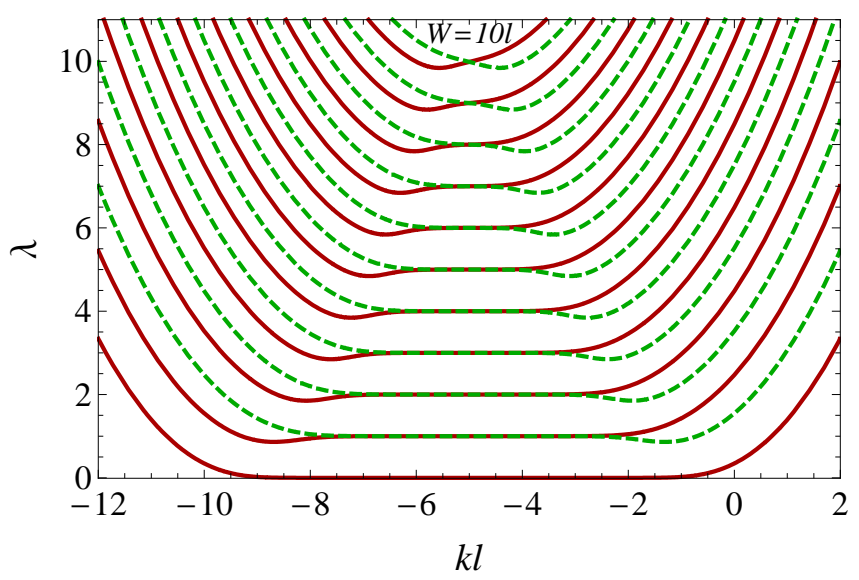

FIG. 6: (Color online) Numerical solutions of Eq. (49) for the dimensionless parameter $\lambda=\left[(E+\mu)^{2}-\tilde{\Delta}^{2}\right] / \epsilon_{0}^{2}$ in the case of armchair edges when $\tilde{\mu}=\Delta=0$. In the higher Landau levels, $n \geq 1$, the solutions to the equations $f_{-}(k, \lambda)=0$ and $f_{+}(k, \lambda)=0$ are shown by solid and dashed lines, respectively.
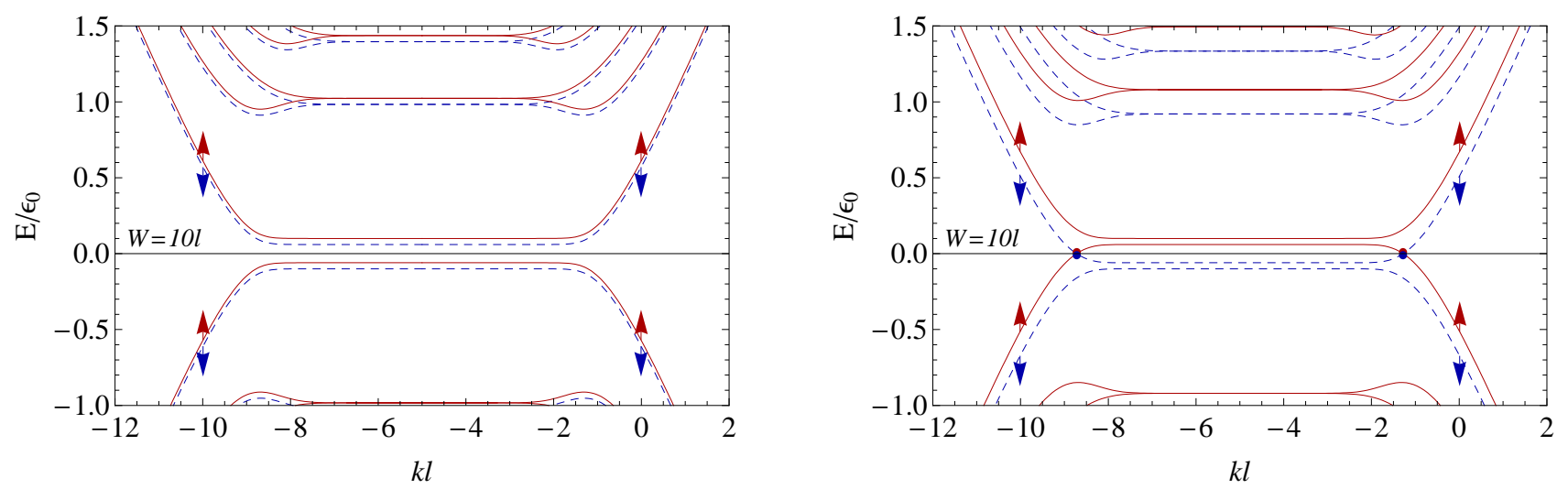

FIG. 7: (Color online) Same as Fig. 5 but for the case of nonzero triplet masses. Nonzero dynamical parameters are as follows: $\mu_{ \pm}=\mp 0.02 \epsilon_{0}, \tilde{\Delta}_{ \pm}=0.08 \epsilon_{0}$ (left panel) and $\mu_{ \pm}=\mp 0.08 \epsilon_{0}, \tilde{\Delta}_{ \pm}=0.02 \epsilon_{0}$ (right panel). The existence of gapless modes (marked by dots) depends on the relative magnitude of $\left|\mu_{ \pm}\right|$and $\left|\tilde{\Delta}_{ \pm}\right|$. The electron spins of the lowest energy sublevels are marked by arrows.

numerical approaches that allows to get a deeper insight into the nature of edge states.

In the case of zigzag edges, gapless modes exist when the ferromagnetic (Zeeman-like) gap dominates over the mass gap of any type, or, more formally, when any of the conditions $\left|\mu_{s}^{( \pm)}\right|>\left|\Delta_{s}^{(\mp)}\right|$ are satisfied for at least one spin choice $s= \pm$. This is consistent with the two limiting cases analyzed in Ref. 15 .

For a ribbon with armchair edges, the condition for the existence of gapless modes is more involved however. In this case, it depends on the actual type (singlet or triplet) of the dynamical Dirac mass induced. For singlet Dirac masses, there are always exist gapless modes. On the other hand, for triplet masses, gapless modes exist only when there is a sufficiently large ferromagnetic (Zeeman-like) gap that dominates over the masses.

One of the most interesting consequences of our finding here is a possibility of resolving the seemingly contradicting interpretations of the $\nu=0$ plateau in terms of either quantum Hall metal or insulator regimes. ${ }^{15,16}$ As follows from Eq. (11), the criterion for the existence of gapless edge states at the Dirac neutral point takes the simple form $Z+A>M$ (note that since a boundary of real graphene samples consists of both zigzag and armchair edges $\stackrel{2}{,}$ it is appropriate to use the more constrained condition for the existence of gapless modes corresponding to the zigzag edges). As was pointed in Refs. 31,32, this condition implies the existence of a critical value of the transverse magnetic field $B^{(c r)}$, where the insulator regime switches to the metallic one. A crude estimate for this value yields $0.01 \mathrm{~T} \lesssim B^{(c r)} \lesssim 200 \mathrm{~T}$ for an effective coupling constant $\lambda$ taken in the interval $0.02 \lesssim \lambda \lesssim 0.2$.32 As one can see, $B^{(c r)}$ is very sensitive to the choice of $\lambda$. In order to fix the values of $\lambda$ and $B^{(c r)}$ more accurately, one should utilize 
more realistic models of graphene that incorporate consistently disorder among other things $\underline{45,46}$ This is a topic for future studies however.

There is another possibility to explain the experimental results in Ref. 16. As was shown in Ref. 32, besides the $S 1$ solution (11), there is another, triplet $(T)$, solution around the Dirac neutral point corresponding to the $\nu=0$ plateau, in the model of Refs. 31,32. In the $T$ solution, while both spin up and spin down quasiparticle states have a triplet Dirac mass $\tilde{\Delta}_{ \pm}=M$, the chemical potentials $\mu_{ \pm}$are small, $\mu_{ \pm}=\mp Z$. Therefore, there are no gapless edge states for this solution and it describes the quantum Hall insulator regime. Calculating the difference of the free energy densities for these two solutions, it was shown ${ }^{32}$ that it is the Zeeman term which makes the $S 1$ solution more favorable: without it, the $S 1$ and $T$ solutions would correspond to two degenerate ground states. On the other hand, as was pointed out in Ref. 20 (see also Refs. 25, 47, 48), there are small on-site repulsion interaction terms on the graphene lattice which favor the triplet solution (such terms were ignored in the model in Refs. 31,32). It would be interesting to figure out the role of these terms in choosing the genuine ground state in the present dynamics at different values of a magnetic field.

In the future, it would be interesting to extend the present analysis by considering inhomogeneous QHF and MC order parameters, which should be consistently determined from the gap equation. Such inhomogeneous order parameters on a ribbon with zigzag edges in a magnetic field can be expected because they exist on ribbons with the zigzag edges in the absence of a magnetic field. 35,49,50,51 The point is that as a consequence of the nearly dispersionless (flat) subbands, a peak in the density of states occurs near the zigzag edge, resulting in an ordered magnetic phase even at zero magnetic field ${ }^{35}$ In the presence of a strong magnetic field, however, the MC and QHF order parameters alone remove the degeneracy of the dispersionless states with opposite spins (or pseudospins, related to the $S U(2)_{s}$ symmetries discussed in Sec. III). This means that the edge magnetism (or pseudomagnetism) may at least partially be captured by the homogeneous order parameters already included in this paper. Of course, it would be important to reexamine these arguments in more detail in future studies.

\section{Acknowledgments}

The authors acknowledge discussions with E. Gorbar, H. Fertig, I. Herbut, L. Levitov, and B. Shklovskii. The work of V.P.G. was supported by the SCOPES-project IB 7320-110848 of the Swiss NSF, the grant 10/07-N "Nanostructure systems, nanomaterials, nanotechnologies", and by the Program of Fundamental Research of the Physics and Astronomy Division of the National Academy of Sciences of Ukraine. V.A.M. and C.M.W. acknowledge the support of the Natural Sciences and Engineering Research Council of Canada. The work of S.G.S. was supported by the Program of Fundamental Research of the Physics and Astronomy Division of the National Academy of Sciences of Ukraine. The work of I.A.S. was supported by the start-up funds from the School of Applied Arts and Sciences at the Arizona State University. This work was made possible by the facilities of the Shared Hierarchical Academic Research Computing Network (SHARCNET:www.sharcnet.ca).

* On leave from Bogolyubov Institute for Theoretical Physics, 03680, Kiev, Ukraine

1 K.S. Novoselov, A.K. Geim, S.V. Morozov, D. Jiang, Y. Zhang, S.V. Dubonos, I.V. Grigorieva, and A.A. Firsov, Science 306, 666 (2004).

2 A.K. Geim and K.S. Novoselov, Nat. Mater. 6, 183 (2007).

3 M. I. Katsnelson and K. S. Novoselov, Solid State Comm. 143, 3 (2007).

4 A. H. Castro Neto, F. Guinea, N. M. R. Peres, K. S. Novoselov and A. K. Geim, Rev. Mod. Phys. 81, 109 (2009).

5 V. P. Gusynin, S. G. Sharapov, and J. P. Carbotte, Int. J. of Mod. Phys. B 21, 4611 (2007).

${ }^{6}$ K. S. Novoselov, A. K. Geim, S. V. Morozov, D. Jaing, M. I. Katsnelson, I. V. Grigorieva, S. V. Dubonos, and A. A. Firsov, Nature 438, 197 (2005).

7 Y. Zhang, Y.-W. Tan, H. L. Störmer, and P. Kim, Nature 438, 201 (2005).

8 Y. Zheng and T. Ando, Phys. Rev. B 65, 245420 (2002).

9 V. P. Gusynin and S. G. Sharapov, Phys. Rev. Lett. 95, 146801 (2005); Phys. Rev. B 73, 245411 (2006).

10 N. M. R. Peres, F. Guinea, and A. H. Castro Neto, Phys. Rev. B 73, 125411 (2006).

11 G. W. Semenoff, Phys. Rev. Lett. 53, 2449 (1984).

12 F. D. M. Haldane, Phys. Rev. Lett. 61, 2015 (1988).

13 Y. Zhang, Z. Jiang, J. P. Small, M. S. Purewal, Y.-W. Tan, M. Fazlollahi, J. D. Chudow, J. A. Jaszczak, H. L. Störmer, and P. Kim, Phys. Rev. Lett. 96, 136806 (2006).

14 Z. Jiang, Y. Zhang, H. L. Störmer, and P. Kim Phys. Rev. Lett. 99, 106802 (2007).

15 D.A. Abanin, K.S. Novoselov, U. Zeitler, P.A. Lee, A.K. Geim, and L.S. Levitov, Phys. Rev. Lett. 98, 196806 (2007).

16 J. G. Checkelsky, L. Li, and N. P. Ong, Phys. Rev. Lett. 100, 206801 (2008); arXiv:0808.0906 [cond-mat.mes-hall]. 
17 K. Nomura and A. H. MacDonald, Phys. Rev. Lett. 96, 256602 (2006).

18 K. Yang, S. Das Sarma, and A. H. MacDonald, Phys. Rev. B 74, 075423 (2006).

19 M. O. Goerbig, R. Moessner, and B. Douçot, Phys. Rev. B 74, 161407(R) (2006).

20 J. Alicea and M. P. A. Fisher, Phys. Rev. B 74, 075422 (2006).

${ }^{21}$ L. Sheng, D. N. Sheng, F. D. M. Haldane, and L. Balents, Phys. Rev. Lett. 99, 196802 (2007).

${ }^{22}$ M.M. Fogler and B.I. Shklovskii, Phys. Rev. B 52, 17366 (1995).

23 D. A. Abanin, P. A. Lee, and L. S. Levitov, Phys. Rev. Lett. 96, 176803 (2006); Solid State Comm. 143, 77 (2007).

24 V. P. Gusynin, V. A. Miransky, S. G. Sharapov, and I. A. Shovkovy, Phys. Rev. B 74, 195429 (2006).

25 I. F. Herbut, Phys. Rev. Lett. 97, 146401 (2006); Phys. Rev. B 75, 165411 (2007); ibid. 76, 085432 (2007).

26 J.-N. Fuchs and P. Lederer, Phys. Rev. Lett. 98, 016803 (2007).

27 M. Ezawa, J. Phys. Soc. Jpn. 76, 094701 (2007).

28 V. P. Gusynin, V. A. Miransky, and I. A. Shovkovy, Phys. Rev. Lett. 73, 3499 (1994); Phys. Rev. D 52, 4718 (1995).

29 D. V. Khveshchenko, Phys. Rev. Lett. 87, 206401 (2001); ibid. 87, 246802 (2001); D. V. Khveshchenko and H. Leal, Nucl.Phys. 687, 323 (2004).

30 E. V. Gorbar, V. P. Gusynin, V. A. Miransky, and I. A. Shovkovy, Phys. Rev. B 66, 045108 (2002); Phys. Lett. A 313, 472 (2003).

31 E. V. Gorbar, V.P. Gusynin, and V. A. Miransky, Low Temp. Phys. 34, 790 (2008).

32 E. V. Gorbar, V.P. Gusynin, V. A. Miransky, and I. A. Shovkovy, Phys. Rev. B 78, 085437 (2008).

33 B. I. Halperin, Phys. Rev. B 25, 2185 (1982).

34 K. Nakada, M. Fujita, G. Dresselhaus, and M. S. Dresselhaus, Phys. Rev. B 54, 17954 (1996).

35 M. Fujita, K. Wakabayashi, K. Nakada, and K. Kusakabe, J. Phys. Soc. Japan 65, 1920 (1996).

36 E. McCann and V. I. Fal'ko, J. Phys.: Condens. Matter 16, 2371 (2004).

37 C. L. Kane and E. J. Mele, Phys. Rev. Lett. 95, 146802 (2005); ibid. 95, 226801 (2005).

38 L. Brey and H. A. Fertig, Phys. Rev. B 73, 195408 (2006); H. A. Fertig and L. Brey, Phys. Rev. Lett. 97, 116805 (2006).

${ }^{39}$ L. Brey and H. A. Fertig, Phys. Rev. B 73, 235411 (2006).

40 N. M. R. Peres, A. H. Castro Neto, and F. Guinea, Phys. Rev. B 73, 241403(R) (2006).

41 J. Milton Pereira, F. M. Peeters, and P. Vasilopoulos, Phys. Rev. B 75, 125433 (2007).

42 V. P. Gusynin, V. A. Miransky, S. G. Sharapov, and I. A. Shovkovy, Phys. Rev. B 77, 205409 (2008).

43 M. Arikawa, Y. Hatsugai, and H. Aoki, Phys. Rev. B 78, 205401 (2008).

44 M. Abramowitz and I. A. Stegun, Handbook of Mathematical Functions With Formulas, Graphs, and Mathematical Tables (U. S. Goverment Printing Office, Washington D.C., 1972) p. 685.

45 P. Goswami, X. Jia, and S. Chakravarty, Phys. Rev. B 76, 205408 (2007); X. Jia, P. Goswami, and S. Chakravarty, Phys. Rev. Lett. 101, 036805 (2008).

${ }^{46}$ K. Nomura, S. Ryu, M. Koshino, C. Mudry, and A. Furusaki, Phys. Rev. Lett. 100, 246806 (2008).

47 I. L. Aleiner, D. E. Kharzeev, and A. M. Tsvelik, Phys. Rev. B 76, 195415 (2007).

${ }^{48}$ I. F. Herbut, V. Juricic, and B. Roy, arXiv:0811.0610 [cond-mat.str-el].

49 A. Yamashiro, Y. Shimoi, K. Harigaya, K. Wakabayashi, Phys. Rev. B 68, 193410 (2003).

50 J. Fernández-Rossier, Phys. Rev. B 77, 075430 (2008).

51 J. Jung, T. Pereg-Barnea, A. H. MacDonald, arXiv:0812.1047 [cond-mat.mes-hall]. 